\title{
GINCANA ANATÔMICA - METODOLOGIA ATIVA no ensino-aprendizagem da Medicina Veterinária: relato de caso
}

\section{Anatomy competition - active methodology in teaching- learning of Veterinary Medicine: case report}

\author{
Airton Renan Bastos Soares ${ }^{1 *} \mathbb{D}^{\text {, Suzana Mourão Gomes }}{ }^{1}$, Luana Brito Bluhm de Bastos ${ }^{1}$, \\ Natália Rodrigues Marques², Adriana Novaes dos Reis ${ }^{3}$ \\ *Autor Correspondente: Airton Renan Bastos Soares. Instituto de Medicina Veterinária, \\ Universidade Federal do Pará, Campus II, Av. Br 316, Km 62, \\ Castanhal, Pará, Brasil. CEP: 68743-970. \\ E-mail: airtonrsoares@gmail.com
}

Como citar: SOARES, A. R. B. et al., Gincana anatômica - metodologia ativa no ensino-aprendizagem da Medicina Veterinária: relato de caso. Revista de Educação Continuada em Medicina Veterinária e Zootecnia do CRMV-SP, São Paulo, v. 19, n. 1, 2021, e38039. DOl: https://doi.org/10.36440/recmvz.v19i1.38039.

Cite as: SOARES, A. R. B. et al., Anatomy Competition - Active Methodology In Teaching-Learning In The Veterinary Medicine: case report. Journal of Continuing Education in Veterinary Medicine and Animal

Science of CRMV-SP, São Paulo, v. 19, n. 1, 2021, e38039. DOl: https://doi.org/10.36440/recmvz.v19i1.38039.

\section{Resumo}

0 presente trabalho descreve uma metodologia ativa para auxiliar o ensino-aprendizado na formação de profissionais mais capacitados e contribuir como modelo de estudo para alunos da disciplina de Anatomia Veterinária. A gincana foi realizada com a participação de alunos $(n=48)$ do primeiro semestre da Faculdade de Medicina Veterinária da Universidade Federal do Pará - Castanhal, no ano de 2018. A turma foi dividida nos grupos A e B, com 24 discentes em cada. 0 docente (mediador) informou as regras da atividade. Os representantes do grupo $A$ foram alocados em cadeiras individuais de no 1 a 24 em um lado da sala, o mesmo ocorreu para o grupo B, no lado oposto. A atividade foi iniciada pela dupla da cadeira no 1 por grupo e finalizada pela dupla da cadeira no 24 . Cada dupla foi chamada pelo mediador da atividade a sentar nos dois bancos no centro da sala, e então foi realizado o 'par ou ímpar' com cada dupla para o vencedor escolher um balão pregado na parede da sala, estourar e direcionar o papel que estava no seu interior com as instruções da atividade a ser proposta pelo mediador. As atividades consistiam em 1 ) responder as perguntas, 2) realizar mímica e 3) participar da "caixa misteriosa". As pontuações foram acerto (+) ou erro (-), conforme critérios preestabelecidos

1 Discente da Faculdade de Medicina Veterinária, Universidade Federal do Pará, Castanhal, Pará, Brasil

2 Técnica do Laboratório de Anatomia dos Animais Domésticos, Faculdade de Medicina Veterinária, Universidade Federal do Pará, Castanhal, Pará, Brasil

3 Docente do Laboratório de Anatomia dos Animais Domésticos, Faculdade de Medicina Veterinária, Universidade Federal do Pará, Castanhal, Pará, Brasil 
no papel no interior do balão. A utilização de MA despertou a curiosidade dos alunos, exigindo que eles adquirissem mais conhecimento para poder participar da atividade e apesar de haver restrições na análise de sua efetividade por se tratar apenas de aspectos extraídos da fala dos estudantes, a dinâmica educacional auxiliou no desenvolvimento de competências técnicas e humanísticas. A experiência realizada destaca a importância da difusão de técnicas ativas de ensino, especialmente, em instituições de ensino superior.

Palavras-chave: Modelo de estudo. Dinâmica educacional. Anatomia Veterinária.

\section{Abstract}

The aim of this study was the description of an active learning that can assist the teachinglearning process (TLP) in the formation of more qualified professionals, and to contribute as a study model for students of Veterinary Anatomy. A total of 48 first year students from the Faculty of Veterinary Medicine (Universidade Federal do Pará/Castanhal) participated in the game. The class was divided into groups $A$ and $B$, with 24 students in each of them. The teacher (mediator) informed the rules of the activity. The representatives of group $A$ were allocated individual chairs from number 1 to 24 on one side of the room, and the same occurred for group B on the opposite side. Each pair was called by the mediator to sit on two benches in the center of the room, and then a game of "odd or even" was played with each pair so that the winner could choose a balloon stuck on the wall of the room, pop it, and send the paper with the instructions of the activity to the mediator. The activities consisted of 1) answering the questions, 2) mimic, and 3) participate in the "mystery box". The scores were hit (+) or error (-) according to pre-established criteria. The use of TLP aroused the students' curiosity, demanding that they acquire more knowledge in order to participate in the activity. And although there are restrictions in the analysis of its effectiveness because it deals only with aspects extracted from the students' speech, the educational dynamics helped in the development of technical and humanistic skills. Thus, the practice applied showed the importance of active learning techniques dissemination, mainly in higher education institutions.

Keywords: Study model. Educational dynamics. Veterinary Anatomy.

\section{Introdução}

Dentre as ciências básicas, a anatomia animal estuda as estruturas macroscópicas do corpo (QUEIRÓZ, 2005), principalmente, em cadáveres submetidos à dissecação (KENNEDY; OLSON, 2009). Ela é uma disciplina indispensável para a vida acadêmica, pois diversas terminologias da Medicina Veterinária fazem parte do seu conteúdo (PAWLINA; LACHMAN, 2004). Contudo, o seu ensino tradicional remete à realização de aulas expositivas dissociadas das práticas (ARAÚJO JÚNIOR et al., 2014), o que dificulta a compreensão do estudante e passa a ser um trabalho árduo que exige a memorização de uma grande quantidade de estruturas (DYCE; SACK; WENSING, 1997) com complexa nomenclatura (MORAES; SCHWINGEL; SILVA JÚNIOR, 2016).

$\mathrm{Na}$ atualidade, na Medicina Veterinária novos processos têm sido empregados para a formação e capacitação de profissionais, preparados para trabalhar em grupo. Dentre eles, destaca-se o emprego de práticas metodológicas modernas que tornam os professores apenas incentivadores da aprendizagem (DA SILVA et al., 2016). Esses procedimentos, contribuem para a formação de cidadãos curiosos e críticos, com vontade de aprender e que acompanham o desenvolvimento científico e tecnológico da sociedade (CAZELLI; MARANDINO; STUDART, 2003). Tais recursos oferecem uma forma mais dinâmica para repassar as informações, facilitam o processo de ensino-aprendizagem e promovem a participação ativa dos estudantes (DIESEL; BALDEZ; MARTINS, 2017), que passam a ter uma visão generalizada e interdisciplinar dos conteúdos ministrados (MITRE et al., 2008). 
0 desenvolvimento de um novo pensamento pedagógico de ensino-aprendizagem, fez com que monitorar passasse a ser a palavra da vez das metodologias ativas (MA), (PEIXOTO, 2016). De fato, neste processo o discente auxilia o docente na transferência de conteúdos da área em estudo e também se capacita desenvolvendo atividades inerentes à docência, aprofundando os seus conhecimentos e solucionando dificuldades que possam surgir durante a aula (MITRE et al., 2008). Assim o professor e o monitor adotam novas formas de aprendizagem que integram: teoria, prática, ensino e serviço (HAAG et al., 2008).

A nova visão de ensino integrado, capacita o estudante para desenvolver o comportamento de autonomia frente às adversidades enfrentadas (VIGNOCHI et al., 2009), e também estimula a adoção de mudanças do seu comportamento frente ao estudo, que o tornam mais autoconfiante (MORAIS; MANZINI, 2006). Na metodologia proposta os papéis, tanto do professor quanto do aluno, passam a ser fundamentais durante todo o processo de ensino aprendizagem, a partir do momento em que assumem atribuições distintas (CARDINOT et al., 2014).

Dentre as MA que contrastam com os métodos tradicionais de ensino-aprendizagem, destacase a Aprendizagem Baseada em Problemas (ABP), em que os alunos adquirem o conhecimento por meio de solução colaborativa de desafios (MARIN et al., 2010; SOBRAL; CAMPOS, 2012), desenvolvem o comportamento de autonomia frente às adversidades enfrentadas (VIGNOCHI et al., 2009) e são estimulados a adotar mudanças frente ao estudo, tornando-se mais autoconfiantes (MORAIS; MANZINI, 2006). Em tal processo, os papéis do professor e do aluno são fundamentais, pois assumem atribuições distintas (SEMIM; SOUZA, 2009). No ano de 2012, o Conselho Federal de Medicina Veterinária, em consenso com as Diretrizes Curriculares do Curso de Medicina Veterinária, lançou um documento intitulado: "Estratégias de ensino-aprendizagem para o desenvolvimento das competências humanísticas". Esse documento descreve diversas técnicas de metodologias ativas destinadas a auxiliar os docentes na construção de um processo que integre adequadamente o discente como sujeito de seu aprendizado, bem como no desenvolvimento de competências humanísticas inerentes ao profissional médico-veterinário.

O presente trabalho descreve a experiência do emprego de uma metodologia ativa de ensino na disciplina de Anatomia Animal na forma de gincana para alunos da Medicina Veterinária, destinada a auxiliar o ensino-aprendizado na formação de profissionais mais capacitados e a contribuir como modelo de atividade para cursos de Medicina Veterinária.

\section{Material e Métodos}

A gincana foi realizada pelos monitores, o docente da disciplina e a técnica de laboratório do Laboratório de Anatomia dos Animais Domésticos (LAAD) com a participação de alunos $(n=48)$ do primeiro semestre da Faculdade de Medicina Veterinária da Universidade Federal do Pará - UFPA/ Castanhal do ano de 2018. Previamente, o docente explicou o fluxo da atividade prática para dirimir qualquer dúvida ou questionamento acerca das regras e resultado final (Figura 1).

Figura 1: Fluxograma da gincana de Anatomia dos Animais Domésticos

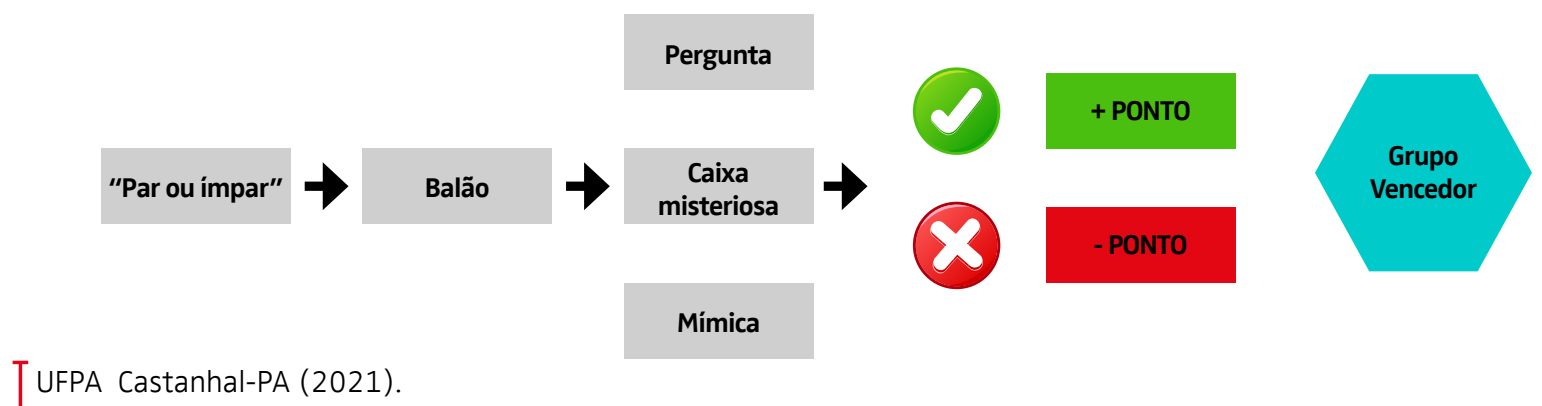


Os discentes foram divididos nos grupos $A(n=24)$ e $B(n=24)$, separados no interior de sua sala em lados opostos e os componentes dos grupos foram distribuídos em cadeiras paralelas do no 1 ao 24. No centro da sala havia uma mesa, duas cadeiras e uma campainha de mesa. Em uma das paredes foram anexados 50 balões de festa de cinco cores (azul, amarelo, verde, branco e rosa), contendo um papel em seu interior com informações acerca da dinâmica que os grupos teriam que desenvolver: (1) mímica de uma determinada estrutura; (2) pergunta e (3) "caixa misteriosa". No outro lado da parede ficou o quadro para os monitores da disciplina anotarem e somarem as pontuações por grupo (Figura 2). As pontuações das equipes foram distribuídas conforme critérios previamente estabelecidos que consistiam na pontuação da dinâmica descrita no papel (acerto [+] ou erro [-]), de acordo com o grau de dificuldade: zero ponto (fácil); um ponto (média); e dois pontos (difícil). A gincana foi realizada por três horas, em três séries de 50 min., com intervalo de 10 min. entre cada série para o descanso dos alunos. Os monitores da disciplina participaram com a sugestão de perguntas, organização da sala de aula, auxilio ao mediador na dinâmica e anotando os pontos das equipes no quadro magnético para que todos os participantes tivessem acesso à evolução da competição.

Figura 2: Layout da sala de aula para a gincana de ensino/aprendizagem na disciplina de Anatomia dos Animais Doméstico

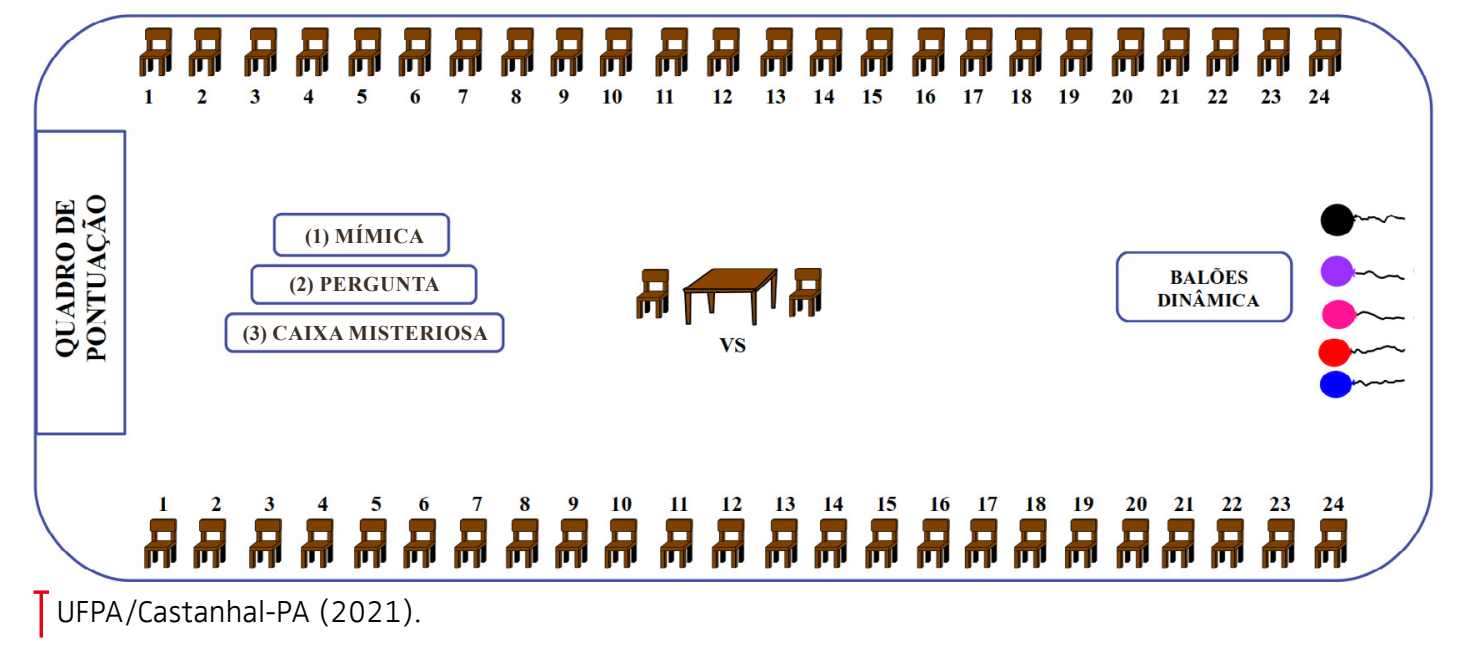

A atividade foi iniciada, por grupo, pela dupla na cadeira no 1 e finalizou com a dupla da cadeira no 24. Cada dupla foi chamada pelo mediador da atividade (docente da disciplina) a sentar nos bancos do centro. Foi sempre realizado o 'par ou ímpar' com cada dupla para que o vencedor escolhesse um balão, o estourasse e encaminhasse o papel com as instruções para o mediador.

Dinâmica 1 (pergunta): os alunos deveriam elevar a mão direita na orelha ipsilateral para ouvir a pergunta e após o mediador ler a pergunta, caso estivessem aptos a respondê-la, deveriam tocar a campainha e quem a tocasse primeiro, deveria apresentar a resposta correta em até 1 min., quando a resposta era incorreta, a oportunidade era passada ao concorrente.

Dinâmica 2 (caixa misteriosa): o discente que escolhia o balão com essa dinâmica, direcionavase à uma caixa retangular de papelão com dois furos laterais, que continha uma peça osteológica de um animal escolhido pelos monitores. Nesta dinâmica, o aluno deveria referir corretamente o nome da peça palpada em até um minuto.

Dinâmica 3 (mímica): o participante que escolheu o respectivo balão, posicionava-se no centro da sala de frente para o grupo e por livre escolha realizava uma série de movimentos (gestos ou sinais), em um minuto, para que o grupo descobrisse o que estava escrito no balão.

Ao final da tarefa, foi realizada a contabilização dos pontos (Total = acerto - erro) e a equipe vencedora foi a que obteve a maior pontuação. A equipe vencedora e a concorrente foram premiadas com dois e um ponto extra, respectivamente, em sua próxima avaliação da disciplina. 


\section{Resultados e discussão}

A utilização de metodologias ativas como o processo de gincana descrito no presente trabalho, despertou a curiosidade dos alunos, fez com que estudassem, se esforçassem e trabalhassem em grupo, desempenhando um papel significativo no processo de ensino-aprendizagem. Salbego et al., (2015), destacam a necessidade da mudança na conduta dos professores, com a adoção de ações que estimulem a interação e o interesse dos alunos, influenciando positivamente todo o processo de formação (CASTOLDI; POLINARSKI, 2009). Este novo formato de aula, baseado em atividades interativas que despertem a criatividade dos alunos, possibilita a aproximação dos participantes envolvidos e 0 interesse pelo trabalho em equipe (MORAIS; MANZINI, 2006).

Os conteúdos estudados coletivamente em cada grupo, possibilitaram a ampliação do conhecimento e reduziram a educação fragmentada. Essa proposta está de acordo com a ABP, em que por meio de uma dinâmica, o docente desperta a capacidade do discente resolver problemas (FARIAS; MARTIN; CRISTO; BERBEL, 2017). Silva (2015), analisando a relação dos estudantes da área de saúde com as MA, verificou que eles a definem como um instrumento de otimização em seu processo de aprendizagem e autonomia. Essas metodologias são essenciais, especialmente no ensino de técnicas consideradas invasivas (OLIVEIRA; FARIA, 2019). Por conseguinte, é preciso destacar a importância da sua difusão nas Instituições de Ensino Superior (IES). De fato, desde a década de 1990, o Conselho Federal de Medicina Veterinária realiza o Seminário Nacional de Educação da Medicina Veterinária, destinado a promover a discussão de assuntos pertinentes ao modelo de ensino da profissão no País, pois a construção dos profissionais médicosveterinários deve ocorrer de maneira conjunta as exigências da sociedade contemporânea e pressentir as demandas futuras. Para que tais objetivos sejam atingidos, a formação de docentes capacitados é um fator determinante (TORRES; CHIRELLI, 2019).

Paiva et al., (2016) observaram que as metodologias ativas têm sido utilizadas da educação básica até a superior, com uma maior frequência em cursos vinculados às áreas da saúde, contribuindo para a desconstrução do ensino tradicional e auxiliando na formação crítica e reflexiva do discente. De fato, as MA têm sido utilizadas em diversas instituições do mundo e a despeito de apresentarem limitações, elas possibilitam a obtenção de resultados promissores na autonomia do educando (FARIAS et al., 2015).

A dinâmica realizada no presente trabalho foi executada após a realização das aulas expositivas, fazendo com que a atividade fosse um meio de otimização e fixação dos conteúdos ministrados, contribuindo assim para que o discente atuasse como a "peça central" do seu aprendizado. Dessa forma, o processamento cognitivo para obtenção de um aprendizado significativo, ocorre a partir do conhecimento prévio do estudante e o conduz a escolha por aprender (AUSUBEL, 2000).

Gomes (2019), descreveu que no curso de Medicina Veterinária da Universidade Federal do Pará, Castanhal (Pará), 82,2 \% dos discentes abordados consideram que o emprego de MA é benéfico para aprendizagem, sendo que $84 \%$ afirmaram que este processo é favorecido quando é associado as metodologias tradicionais de ensino. Pfrimer e Melo (2020) afirmam que as MA são recursos importantes para o estudo da anatomia animal, contudo, os métodos tradicionais ainda são indispensáveis.

Em relação a monitoria acadêmica, Cardinot et al., (2014) dizem que a interação de alunosmonitores com alunos da disciplina de anatomia possibilita a interatividade entre alunos de diferentes turmas e melhora a fixação dos conteúdos. Além disso, a monitoria acadêmica é positiva para alunos da disciplina de anatomia (CALAZANS, 2013).

O contexto acadêmico é estressante e desgastante, especialmente, para quem não possui equilíbrio emocional, sem considerar os desafios diários (metas, prazos, família, relacionamentos, saúde e problemas financeiros). No presente trabalho, os estudantes relataram que a metodologia adotada foi de grande ajuda, pois estavam com dificuldades em aprender o assunto ministrado na forma tradicional, adquirindo competência, ou seja, a capacidade de combinar conhecimentos, habilidades e atitudes. A dinâmica realizada contribuiu para o aprendizado, possibilitou a diversão 
e o desenvolvimento de habilidades (autoconhecimento emocional, controle emocional, empatia e habilidades sociais), vinculadas à inteligência emocional, como forma de se alcançar a satisfação em termos gerais (GOLEMAN, 1995). Valle et al., (2014) concluíram que a inteligência emocional está positivamente vinculada à capacidade de empreendedorismo e que possibilita o desenvolvimento de características emocionais necessárias não só para o desempenho acadêmico, mas também para a formação de indivíduos mais aptos ao mercado de trabalho.

\section{Conclusão}

O emprego de uma metodologia ativa, no formato de gincana anatômica, propiciou o desenvolvimento de competências técnicas e humanísticas nos alunos, pois desencadeou uma dinâmica bastante participativa, motivada pela competição estabelecida entre os grupos, com a discussão dos assuntos pertinentes ao conteúdo programático da disciplina. A estratégia da atividade aplicada estimulou a criatividade dos alunos e proporcionou o aprendizado de assuntos técnicos de forma agradável e participativa, confirmando assim a importância da difusão de técnicas ativas de ensino, especialmente em instituições de ensino superior.\&

\section{Referências}

ARAÚJO JÚNIOR, J. P. et al., Desafio anatômico: uma metodologia capaz de auxiliar no aprendizado de anatomia humana. Medicina, Ribeirão Preto, ajustar os espaçamentos: v. 47, n. 1, p. 62- 68, 2014. DOI: https://doi.org/10.11606/issn.2176-7262.v47i1p62-68.

AUSUBEL, D. P. The acquisition and retention of knowledge: a cognitive view. Boston: Kluwer Academic Publishers, 2000.

BERBEL, N. A. N. As metodologias ativas e a promoção da autonomia de estudantes. Seminário de Ciências Sociais e Humanas, v. 32, n. 1, p. 25-40, jan. - jun. 2011.

CALAZANS, N. C. 0 ensino e o aprendizado práticos da anatomia humana: Uma revisão de literatura. 2013. Monografia (Conclusão de Curso) - Universidade Federal da Bahia, Faculdade de Medicina Veterinária da Bahia, Salvador, 2013.

CARDINOT, T. M. et al., Importância da disciplina de anatomia humana para os discentes de educação física e fisioterapia da ABEU centro universitário de Belford Roxo/RJ. Coleção Pesquisa em Educação Física, Várzea Paulista/SP, v. 13, n. 1, p. 95-102, 2014.

CASTOLDI, R.; POLINARSKI, C. A. A. Utilização de Recursos Didático-Pedagógicos na Motivação da Aprendizagem. Anais do I Simpósio Nacional de Ensino de Ciência e Tecnologia, p. 684-692, 2009.

CAZELLI, S.; MARANDINO, M.; STUDART, D. C. Educação e Comunicação nos Museus de Ciência: aspectos históricos, pesquisas e prática. In: MARANDINO, M.; GOUVÊA, G.; LEAL, M. C. (Org.). Educação e Museu: a construção social do caráter educativo dos museus de ciência. Rio de Janeiro: Editora Access, p. 83106, 2003.

\section{CONSELHO FEDERAL DE MEDICINA VETERINÁRIA. Estratégias de Ensino-aprendizagem para Desenvolvimento das Competências Humanísticas. 2012, 150 p.}

DA SILVA, C. D. D. et al., Aprendendo sobre o corpo humano: contribuições do pibid para o ensino de ciências. CARPE DIEM: Revista Cultural e Científica do UNIFACEX, v. 14, n. 1, p. 17-30, 2016.

DIESEL, A.; BALDEZ, A. L. S.; MARTINS, S. N. Os princípios das metodologias ativas de ensino: uma 
abordagem teórica. Revista Thema, v. 14, n. 1, p. 268-288, 2017. DOI: https://doi.org/10.15536/ thema.14.2017.268-288.404.

DYCE, K. M.; SACK, W. O.; WENSING, C. J. G. Tratado de Anatomia Veterinária. 2. ed. Rio de Janeiro: Guanabara Koogan, p. 663, 1997.

FARIAS, P. A. M. de; MARTIN, A. L. A. R.; CRISTO, C. S. Aprendizagem Ativa na Educação em Saúde: Percurso Histórico e Aplicações. Revista Brasileira de Educação Médica, v. 39, n. 1, p. 143-50, 2015. DOI: https://doi.org/10.1590/1981-52712015v39n1e00602014.

GOLEMAN, D. Inteligência emocional: a teoria revolucionária que redefine o que é ser inteligente. Bantam Books, 1995.

GOMES, S. M. Percepção dos estudantes de Medicina Veterinária da Universidade Federal do Pará frente às metodologias ativas. In: RUIZ, V. R. R. R. (org.) Estudos em Patologia Veterinária, Ponta Grossa, PR: Atena Editoria, 2019. p. 7 - 14.

HAAG, G. S. et al., Contribuições da monitoria no processo ensino-aprendizagem em enfermagem. Revista Brasileira de Enfermagem. Brasília, v. 61, n. 220, p. 215-220, abr. 2008. DOI: http://dx.doi. org/10.1590/S0034-71672008000200011.

KENNEDY, G. J.; OLSON, T. R. "Cadaver Conference Day": A Psychiatrist in the Gross Anatomy Course. Primary Psychiatry, v. 16, n. 1, p. 26-30, 2009.

MARIN, M. J. S. et al., Aspectos das fortalezas e fragilidades no uso das Metodologias Ativas de Aprendizagem. Revista Brasileira de Educação Médica, v. 34, n. 1, p. 13-20, marc. 2004. D0l: https://doi.org/10.1590/S0100-55022010000100003.

MELO, D. P. G; PFRIMER, G. D. Utilização de massa de modelar em aula prática de anatomia animal no curso de Medicina Veterinária - Relato de experiência. In: VASCONCELOS, A. W. S. (org.) Enfoques Epistemológicos na Formação Docente 2, Ponta Grossa, PR: Atena, 2020. p. 93 - 98. DOI: https://doi. org/10.22533/at.ed.95020140210.

MITRE, S. M. et al., Metodologias ativas de ensino-aprendizagem na formação profissional em saúde: debates atuais. Ciência \& Saúde Coletiva, v. 13, n. 2, p. 2133-2144, dez. 2008. DOI: http://dx.doi. org/10.1590/S1413-81232008000900018.

MORAES, G. N. B.; SCHWINGEL, P. A.; SILVA JÚNIOR, E. X. Uso de roteiros didáticos e modelos anatômicos, alternativos, no ensino-aprendizagem nas aulas práticas de anatomia humana. Revista Ibero-Americana de Estudos em Educação, v. 11, n. 1, p. 223-230, jan. - marc. 2016. DOI: https:// doi.org/10.21723/riaee.2016.v11.n1.p223.

MORAIS, M. A. A.; MANZINI, E. J. Concepções sobre a Aprendizagem Baseada em Problemas: um estudo de caso na Famena. Revista Brasileira de Educação Médica, v. 30, n. 3, p. 125-135, dez. 2006. DOI: https://doi.org/10.1590/S0100-55022006000300003.

OLIVEIRA, G. D. de; FARIA, V. P. Metodologia ativa no treino de sutura: o uso do EVA na construção de maquete de sutura artesanal. Pubvet, v. 13, n. 5, p. 166, 2019. Dol: https://doi.org/10.31533/ pubvet.v13n5a334.1-6.

PAIVA, M. R. F. et al., Metodologias ativas de ensino-aprendizagem: revisão integrativa. SANARERevista de Políticas Públicas, v. 15, n. 2, p. 145-153, jun. - dez. 2016.

PAWLINA, W.; LACHMAN, N. Dissection in Learning and Teaching Gross Anatomy: Rebuttal to McLachlan. The Anatomical Record, v. 281B, n. 1, p. 9-11, nov. 2004. DOI: https://doi.org/10.1002/ ar.b.20038.

PEIXOTO, G. A. O uso de metodologias ativas como ferramenta de potencialização da aprendizagem 
de diagramas de caso de uso. Periódico Científico Outras Palavras, v. 12, n. 2, p. 35, 2016.

QUEIROZ, C. A. F. 0 uso de cadáveres humanos como instrumento na construção de conhecimento a partir de uma visão bioética. 2005. Dissertação (Pós-Graduação em Ciências Ambientais e Saúde) - Universidade Católica de Goiás, Goiânia, 2005.

SALBEGO, C. et al., Percepções acadêmicas sobre o ensino e a aprendizagem em anatomia humana.

Revista Brasileira de Educação Médica, v. 39, n. 1, p. 23-31, jan.-marc. 2015. DOl: https://doi. org/10.1590/1981-52712015v39n1e00732014.

SEMIM, G. M.; SOUZA, M. C. B. M. Professor como facilitador do processo ensino-aprendizagem: visão do estudante de enfermagem. Revista Gaúcha de Enfermagem. Porto Alegre (RS), v. 30, n. 3, p. 484-491, 2009.

SILVA, A. A. Formação em saúde: representações de acadêmicos sobre metodologias ativas de ensino e aprendizagem. Cadernos da Fucamp, v. 14, n. 21, p. 120 - 138, 2015.

SOBRAL, F. R.; CAMPOS, C. J. G. Utilização de metodologia ativa no ensino e assistência de enfermagem na produção nacional: revisão integrativa. Revista da Escola de Enfermagem da USP, v. 46, n. 1, p. 208-218, fev. 2012. DOl: https://doi.org/10.1590/S0080-62342012000100028.

TORRES, V. F.; CHIRELLI, M. Q. Formação docente na Medicina Veterinária: desafios e estratégias desvendados pela análise temática. In: COSTA, P. A. (ed. lit.), SÁ, S. O. (ed. lit.), CASTRO, P. A. (ed.

lit.) Atas - investigação Qualitativa em Educação: 80 Congresso Ibero-Americano em Investigação Qualitativa (CIAIQ2019), Lisboa, Portugal: Ludomedi, 2019. p. 681-690.

VALE, G. M. V.; CORRÊA, V. S.; REIS, R. F. Motivações para o empreendedorismo: necessidade versus oportunidade? Revista de Administração Contemporânea, v. 18, n. 3, p. 311-327, jun. 2014. DOI: http://dx.doi.org/10.1590/1982-7849rac20141612.

VIGNOCHI, C. M. et al., Considerações sobre aprendizagem baseada em problemas na educação em saúde. Artigo de revisão. Revista HCPA, v. 29, n. 1, p. 45-50, 2009. 